\title{
La Hermandad de los Montes de Toledo entre los siglos XIV y XV
}

\author{
José María Sánchez Benito \\ Universidad Autónoma de Madrid
}

\begin{abstract}
RESUMEN
Si la Santa Hermandad Vieja de Toledo, Talavera y Ciudad Real en su conjunto, y

las dos últimas, en particular, han sido objeto de diferentes estudios, aludiendo tanto a la Edad Media como a la Moderna, la rama toledana, por el menor número de fuentes aprovechables para su estudio, aun siendo la principal de las tres ha permanecido en una penumbra que no tiene parangón en el caso de sus dos gemelas. El presente articulo tiene como fin profundizar en sus características, organización y funciones, insistiendo en to que tiene de peculiar, en una cronología -entre los siglos $X I V$ y $X V$ - que resulta especialmente interesante para comprender el proceso evolutivo de esta institución.
\end{abstract}

\section{PALABRAS CLAVE}

Hermandad, colmeneros, Toledo, Montes de Toledo, siglo XIV, siglo XV.

\section{ABSTRACT}

If the Holy Old Brotherhood of Toledo, Talavera and Ciudad Real, considered as a whole, and the last two, in particular, have been the purpose of several studies that mentioned their existence both during the Middle Ages and the Modern Age, the Toledan Branch, although it was the main one of the three, has been almost ignored due to the less number of sources available to its study. This situation is incomparable with its two other twin sisters. The article deals with the study in depth of the features, organization and functions, and emphasizing its peculiarities, during the XIVth and XVth century. This is specially interesting to understand the evolution process of this institution.

\section{KEY WORDS}

Brotherhood, beekeeper, Toledo, Toiedo Mountains, XIVth century, XVth century.

Dejando a un lado las numerosas publicaciones antiguas que de alguna manera hablan acerca de las hermandades viejas de Toledo, Talavera y Ciudad Real, es el artículo clásico del profesor L. Suárez Fernández, titulado "Evolución 
histórica de las hermandades castellanas" ' , el verdadero punto de partida en la investigación tanto de esta clase de instituciones en general como de la que ahora nos ocupa en particular. Con el mismo enfoque global siguió sus huellas años más tarde A. Álvarez de Morales ${ }^{2}$, utilizando una perspectiva evolucionista, y por las mismas fechas M. C. Pescador del Hoyo abordó el estudio monográfico de la triple institución castellano-manchega ${ }^{3}$. Pero ha sido en los últimos veinte años cuando se ha profundizado en su conocimiento de una manera muy importante, analizando primero la etapa medieval y luego la Edad Moderna. En la década de los ochenta dediqué a la Hermandad Vieja mi tesis doctoral, inmediatamente convertida en un libro que planteaba el estudio global de las tres asociaciones ${ }^{4}$, al cual siguieron varios artículos monográficos ${ }^{5}$, mientras que L. R. Villegas Díaz analizaba diversas cuestiones relacionadas específicamente con la hermandad ciudadrealeña ${ }^{6}$. Algo más tarde, ya en los años noventa, salieron a la luz trabajos de relieve centrados en los siglos posteriores, a partir del XVI, y en las entidades que disponían de un mayor número de fuentes documentales, es decir, las de Talavera y Ciudad Real ${ }^{7}$, del mismo modo que dichas fuentes se utilizaban certeramente para profundizar en el análisis de la delincuencia que actuaba en La Mancha entre fines del siglo xv y principios del siguiente ${ }^{8}$. Así pues, resulta in-

Cuadernos de Historia de España, XVI, 1951, pp. 5-78.

2 Las hermandades, expresión del movimiento comunitario en España, Valladolid, 1974.

3 "Los orígenes de la Santa Hermandad", Cuademos de Historia de España, LV-LVI, 1972, pp. $400-43$.

4 Santa Hermandad Vieja de Toledo, Talavera y Ciudad Real (Siglos XII-xV), Toledo, 1987.

5 «Sobre la Hermandad Vieja de Toledo, Talavera y Ciudad Real: conflictos jurisdiccionales y poder sobre la tierra", Anuario de Estudios Medievales, 18. Estudios dedicados a la memoria del prof. Emilio Sáez, 1988, pp. 147-55; "Nuevas observaciones sobre los conflictos entre Ciudad Real y la Orden de Calatrava: el papel de la Hermandad Vieja en los siglos XIV y xV", Revista del Centro Asociado de la U.N.E.D. de Cuenca, 4, 1990-91, pp. 57-73; “Delincuencia y vida rural en La Jara cacereña (1501)», Alcántara, 23-24, 1991, pp. 67-79; «Poder y propiedad. Los hermanos de la Santa Hermandad Vieja de Toledo, Talavera y Ciudad Real en el siglo XV", I Congreso de Historia de Castilla-La Mancha, VI. Campesinos y señores en los siglos XIV y XV, Toledo, 1988, pp. 95-100, y muy recientemente «Hermandades y delincuencia rural entre el Tajo y Sierra Morena", ponencia presentada en el III Coloquio de Historia del Crimen de Durango, 2005.

${ }^{6}$ L. R. VILLEgAs Díaz, «Pobreza y beneficencia en Ciudad Real a fines del reinado de los Reyes Católicos. Las limosnas de la Hermandad Vieja en 1501". Almud, 4, 1981, pp. 199-32; "Sobre la financiación de la Hermandad de Ciudad Real. Sus ingresos (1491-1525)", La ciudad hispánica entre los siglos XIII al XVI. Actas del Coloquio celebrado en La Rábida y Sevilla en septiembre de 1981, Madrid, 1985, pp. 911-28; “La Hermandad de Ciudad Real, instrumento de colonización del territorio», / Congreso de Historia de Castilla-La Mancha, VI. Campesinos y señores en los siglos xIV y xV, Toledo, 1988, pp. 87-93. También A. Romero Martinez y L. R. Villegas Díaz, "Pobreza y beneficencia en ciudad Real: el hospital de la Hermandad Vieja (1491-1525)", Cuadernos de Estudios Medievales, 17, 1992.

7 A. Guillaume-Alonso, Una institución del Antiguo Régimen: La Santa Hermandad Vieja de Talavera de la Reina (Siglos xv' y xvil), Talavera de la Reina, 1995, y M. F. Gómez Vozmediano, La Santa Hermandad Vieja de Ciudad Real en la Edad Moderna. Siglos XVII-xVII, Universidad Complutense de Madrid, tesis doctoral, ed. en CD, 1992. Ambos autores han publicado varios trabajos monográficos que no cito por salirse del ámbito cronológico medieval en el que se centra este artículo y por no alargar demasiado las notas a pie de página. No olvidaré, sin embargo, el trabajo de T. ENGENIOS MARTín, "Aspectos jurisdiccionales de la Santa Hermandad Vieja de Talavera", I Seminario de la Guardia Civil: Pasado, presente y futuro, Aranjuez, 1990, pp. 39-44, y tampoco, por su perspectiva general, el de E. MARTiNEz RUIz y M. F. GómEz VOZMEDIANO, "La jurisdicción de la Hermandad", en Instituciones de la España moderna, 1. Las jurisdicciones, Madrid, 1996, pp. 229-62.

8 J. M. MEndoza GaRRIDO, Violencia, delincuencia y persecución en el Campo de Calatrava a fines de la Edad Media, Ciudad Real, 1995 y sobretodo Delincuencia y represión en la Castilla bajomedieval, Granada, 1999 
dudable que el conocimiento que se tiene acerca de estas instituciones y todo lo que las rodea es al presente copioso e interesante, pues no de otra manera se puede calificar, y desde luego, el avance que se ha producido en el medio siglo largo que nos separa del cimiento puesto por el profesor Suárez es realmente muy grande.

Pero dicho esto, no hay que olvidar que son las ramas de Talavera y Ciudad Real las que han aportado el mayor caudal a este conocimiento y, en cambio, la existente en Toledo, aun siendo la principal, ha quedado hasta ahora en un plano muy secundario, casi diriamos en la oscuridad, porque, al menos en lo referente al medievo, el número de testimonios documentales que arrojaban luz sobre ella es muy escaso. Podemos hacernos una idea de esta dificultad si tenemos en cuenta que cuando en 1990 publiqué mi Colección de documentos de la Santa Hermandad, reuniendo los escritos comprendidos entre los años 1300 y 1500 que por entonces se conocian, tan sólo se referían a los hermanados de Toledo poco más del $8 \%$ del total ${ }^{9}$.

Consecuentemente, este es el sentido del presente artículo, es decir, iluminar el despliegue hermandino en el ámbito toledano, significando sus peculiaridades que no son pocas- $y$ completando así el caudal del conocimiento existente sobre unas asociaciones cuyo impacto en la región que les sirvió de escenario fue muy grande. A tal fin se ha recurrido a la documentación aparecida en los últimos años en el Archivo de la Diputación Provincial de Toledo, convenientemente catalogada por su responsable, M. J. Cruz Arias ${ }^{10}$, y que incluye un libro que en adelante será nuestra principal guía.

En el citado volumen, cuya signatura en el Archivo de la Diputación toledana es Libro $65^{11}$, cabe encontrar anotaciones bastante desordenadas sobre los diferentes asuntos que integraban la vida administrativa de la Hermandad entre los años 1376 y 1418, abarcando un periodo, a caballo entre dos siglos, particularmente interesante para comprender el proceso de construcción y consolidación de la entidad, así como el carácter de su relación con el territorio en el que se desarrollaba. De esta suerte, se puede plantear la cuestión más amplia de la influencia urbana sobre la tierra dependiente de la ciudad.

Se ha dicho innumerables veces que los origenes de las tres hermandades viejas son, en verdad, sumamente oscuros. Como es natural, la escasez de noticias documentales dificulta el esclarecimento de esta incógnita, pero también hay que tener en cuenta que desde hace muy largo tiempo el tema se ha visto ro-

9 J. M. Sánchez Benito, Colección de documentos de la Santa Hermandad (1300-1500), Toledo, 1990.

10 «El fondo documental de la Santa Hermandad Vieja de Toledo en el Archivo de la Diputación Provincial", Archivo Secreto. Revista cultural de Toledo, 1, 2002, pp. 7-46. Quiero agradecerle las facilidades que me ofreció cuando consulté esta documentación en el Archivo.

"Casi toda la información manejada para la realización de este artículo procede de este libro. Por consiguiente, no se volverá a citar en las sucesivas notas a pie de página, al contrario que los datos de otra procedencia, cuyas referencias archivísticas si se indicarán oportunamente. 
deado de un cierto ambiente de leyenda. En el caso concreto de la Hermandad toledana, repasando solamente por encima la literatura escrita sobre esta cuestión - desde la Historia o descripción de la Imperial Ciudad de Toledo publicada por P. Alcocer en 1554 hasta mediados del siglo $x^{12}$ - con facilidad se observan varias cosas. En primer lugar, la búsqueda de antigüedad, intentando llevar el surgimiento de la iniciativa hermandina hasta el siglo XII; en segundo término, el deseo de entroncar precozmente dicha iniciativa con la corona; por último, y como no podía ser de otro modo, el reconocimiento del protagonismo que en esta empresa tuvieron las gentes que desarrollaban actividades económicas en los montes. Para conjugar estos tres factores la pieza esencial era un privilegio real fechado en 1220 por el cual Fernando III favorecía a los cazadores de conejos de la ciudad de Toledo para que pudieran actuar en los mismos territorios que venían utilizando en los tiempos de Alfonso VIII, indicando también que no se lo impidiesen con adehesamientos, y para que, asimismo, mantuviesen los fueros y costumbres que por entonces tenían ${ }^{13}$. Indudablemente, no se menciona en ningún momento ni la Hermandad ni nada que se le parezca, pero desde la perspectiva interesada de quienes querían ennoblecer la institución acrecentando su antigüedad y entroncándola con la corona, este privilegio se utilizó en multitud de ocasiones como el gozne que permitía remontarse hasta el reinado de Alfonso VIII y, ¿por qué no?, hasta su misma minoría, y conectar a los hermanos con este mismo soberano que ya habría otorgado determinados fueros. Insisto en que el documento que estamos comentando no autoriza ninguna de estas hipótesis, cuya base tiene que ver más que nada con la mitificación de la Hermandad, bien visible en los siglos de la Edad Moderna y aún antes.

Con anterioridad he aludido a todas estas versiones de los orígenes hermandinos ${ }^{14}$, por lo que no es mi intención profundizar ahora en toda esta mitificación, baste recordar que en Ciudad Real las explicaciones legendarias adoptan otras variantes; no se aspira aqui a una antigüedad anterior a las Navas de Tolosa, aunque se va más allá de la propia fundación de la ciudad y se personaliza en un caballero supuestamente llamado Gil Turro, al que después habría autorizado expresa-

12 P. Alcocer, Historia o descripción de la Imperial Ciudad de Toledo, Toledo, 1554, fol. CXXIIIr; F. DE PISA, Descripción de la Imperial Ciudad de Toledo, 1617, libro I, capítulo XXIII, fol. 36v; E. TERREROS, Paleografía Española, Madrid, 1757, pp. 54-55; P. SALAZAR DE MEndozA, Monarquía de España, Madrid, 1770, libro III, capítulo XV, pp. 360-61; S. R. PARRO, Toledo en la mano o descripción histórico artistica de la magnifica catedral y los demás célebres monumentos, Toledo, 1857, II, pp. 605-06; C. JIMÉNEZ DE SANDOVAL, Las instituciones de seguridad pública en España y sus dominios de ultramar, Madrid, 1858, p. 76; A. Martín Gamero, Historia de la ciudad de Toledo, Toledo, 1862, II, pp. 833-34; M. Colmeiro, Curso de Derecho Político según la Historia de León y Castilla, Madrid, 1873, pp. 512-13; QUADRADO, J. M. y V. DE LA FUENTE, España, sus monumentos y artes, su naturaleza e historia, Barcelona, 1886, III, p. 401; PALAZUelos, vizconde de, Guía artístico-práctica de Toledo, 1890, p. 1054; A. PAZ y MeliA, "La Santa Real Hermandad Vieja y la Nueva Hermandad General del Reino", Revista de Archivos, Bibliotecas y Museos, 3, 1897, pp. 98-99; C. PALENCIA FloRES, Robos famosos perseguidos por la Santa Hermandad Vieja de Talavera, Toledo, 1981, p. 5 y "Museo de la Santa Hermandad de Toledo", Guias de los museos de España, IX, 1958, pp. 7-8.

13 B.N. mss. 13094, fol. 7, publicado por J. GonzáleZ, Reinado y diplomas de Fernando /I/, Córdoba, 1980, II, n. 112.

14 Ver mi libro Santa Hermandad Vieja..., cit, pp. 42 y ss. 
mente el propio Fernando III ${ }^{15}$. En el caso de Talavera, con menores ambiciones, lo que se hace es localizar una fuerte actividad bandolera en la Sierra de Guadalupe, dando por sentada la existencia de la Hermandad hacia 1214, con el ulterior respaldo de Fernando III $^{16}$.

Pero repito que nada de esto nos interesa demasiado. Para quien trate de estudiar los primeros tiempos de las hermandades solamente aporta confusión y el documento de 1220, único elemento objetivo, nos podrá servir exclusivamente para establecer la existencia de una población que se abre paso en las espesuras de los montes explotando sus recursos, bien sea la caza, que es lo que menciona el documento, o bien otras actividades como la apicultura.

Así pues, y sin entrar a fondo en una temática a la que he dedicado mi atención en otras ocasiones ${ }^{17}$, los primeros pasos de la Hermandad toledana, al igual que sus dos gemelas, deben situarse en un marco delimitado por los factores siguientes:

A La escasa humanización de los montes, explotados de manera muy extensiva por algunos arriesgados que se dedicaban a actividades como la caza o a las colmenas.

B/ La ciudad, que controlaba el territorio, servia de residencia a los propietarios y canalizaba los flujos económicos derivados de la producción montaraz.

C/ Las peripecias de los golfines, impulsadas por la conflictividad política y las algaradas nobiliarias que tanto abundan desde la última fase del reinado de Alfonso $X$, cuyo desarrollo tenía lugar al amparo de una geografía quebrada y solitaria.

De esta manera, durante los últimos años del siglo XIII los que tenían intereses permanentes en los montes - es decir, los propietarios de colmenares especialmente- presionados por las depredaciones de los golfines fueron concertándose para repeler sus agresiones. Lo que hacian no era otra cosa sino poner en práctica el "apellido» en los espacios despoblados, reuniendo cuando habia peligro a las gentes dispersas por las montañas para enfrentarse a los salteadores. Pero no podemos confundirnos, son los propietarios de la ciudad los que articulan este esfuerzo de defensa y terminan consiguiendo la consolidación de la Hermandad.

Ocurre esto a partir de 1300, cuando tanto en Toledo como en Talavera los ciudadanos que estaban impulsando esta iniciativa recurren a los dos municipios

15 J. Diaz JuRAdo, Singular idea del sabio rey don Alonso dibujada en la fundación de Ciudad Real, A.H.P. Ciudad Real manuscrito 3601, pp. 65-66; C. JIMÉNEZ DE SANDOVAL, op. cit., p. 76; J. ZUGASTI, El bandolerismo. Estudio social y memorias históricas, Madrid, 1877, p. 314 y SS; A. BLAZOUEZ Y DELGADOAgullera, Historia de la provincia de Ciudad Real, Ávila, 1898, pp. 10-11; L. Delgado MerCháN, Historia documentada de Ciudad Real, Ciudad Real, 2. ${ }^{a}$ ed., 1907, pp. 288-94.

16 I. Fernandez SÁnchez, Historia de Talavera de la Reina, Talavera de la Reina, 1896, p. 107 y CEDILlo, Conde de, Catálogo monumental de la provincia de Toledo, Toledo, 1958, p. 328.

17 Ver mi libro citado, Santa Hermandad Vieja..., pp. 32-58 y muy recientemente la ponencia presentada en noviembre de 2005 en el III Coloquio del Centro de Historia del Crimen de Durango con el título "Hermandades y delincuencia rural entre el Tajo y Sierra Morena", todavía inédita. 
para obtener su apoyo y legalizar, por decirlo de algún modo, las actividades armadas que estaban llevando a cabo para defenderse de los golfines. La carta toledana se fechó a quince de octubre ${ }^{18}$ y además de reconocer la existencia de la Hermandad, aprueba sus decisiones, de manera que los que estaban en los montes tuvieran armas y acudiesen al ser llamados, del mismo modo que los designados para ser cuadrilleros no pudiesen negarse y los pastores prestasen también su colaboración. En fin, lo que la carta hace es consagrar un mecanismo de movilización que sin duda se estaba empleando en los montes y consistía en la utilización de los que se ganaban la vida entre las espesuras - particularmente en los colmenares-que acudian armados para enfrentarse a los golfines, siendo encuadrados por unos cuadrilleros repartidos por el territorio, cuya tarea consistía en vigilar y reunir a la gente. Por encima de este esquema, y a su frente, figuran los propietarios, de entre los cuales se extraían tres hombres buenos para encabezar la organización. La carta toledana menciona a estos tres hombres buenos por su nombre y reconoce expresamente su función directiva.

Casi inmediatamente, en noviembre del mismo año 1300 , los toledanos y los talaveranos, cada uno con el respaldo de sus respectivos municipios, procedieron a hermanarse, siempre con el mismo fin que es la lucha contra los golfines. Para ello se reunieron en un lugar de los montes, Aliseda de Estena -cuyo nombre será después Navas de Estena-, y elaboraron un ordenamiento, basado lógicamente en las normas que previamente tenian tanto unos como otros ${ }^{19}$. En el mismo, se partía del principio de ayuda mutua, haciéndose saber la presencia de delincuentes y colaborando en lo posible. La mayor atención se dedicaba al orden de la hueste, sancionando, por ejemplo, los insultos o las peleas entre los movilizados. Así es que lo principal seguía siendo la disponibilidad de una fuerza armada fácil de reunir en los montes y como único medio de coordinación entre toledanos y talaveranos se creaba una junta anual que habría de tener lugar en el mismo sitio en el que unos y otros se habían hermanado, es decir, Aliseda de Estena. Finalmente, el proceso de gestación de la Hermandad Vieja de Toledo, Talavera y Villa Real - más tarde Ciudad Real- culmina con la adhesión de la tercera rama, en agosto de 1302 y en el mismo lugar, Aliseda de Estena, mediante un nuevo ordenamiento de todo punto similar al ya vigente entre toledanos y talaveranos ${ }^{20}$.

Muy pronto, en septiembre de 1302 se recibió el primer privilegio real, tras el cual vendrian muchos otros pues no era sino el inicio de una línea constante de

18 B.N. mss. 13030 , fols. 115r-17r, publicada por L. SUÁREZ FERNÁNDEZ, op. cit., pp. 55-57. Hubo una carta similar del concejo de Talavera, dirigida a la hermandad formada en su tierra, actualmente perdida pero que se menciona en otra del mismo concejo, de 27 de junio de 1300, para que se pusieran cuadrilleros de los que vivian en los montes. Nótese que la carta talaverana es anterior a la otorgada por los municipes de Toledo en octubre de 1300 . A. M. Talavera Sta. Hermandad, leg. 23, n. 7, publicada por J. M. SanCHEz Benito, Colección de documentos..., cit., n. 1.

19 Publicado por L. JiMÉNEZ DE LA LLAVE, “La Santa Hermandad de Talavera de la Reina», Boletín de la Real Academia de la Historia., XXII, 1893, pp. 96-99.

20 Publiqué el texto en mi libro Santa Hermandad Vieja..., cit., pp. 313-17, tomándolo del manuscrito de J. Diaz JuRAdo, Singular idea..., cit., fols. 87r-88v. 
respaldo de la monarquía ${ }^{21}$. La triple institución obtenía así unos firmes fundamentos legales y consecuentemente los privilegios otorgados por el trono resultarán absolutamente fundamentales para su prolongada permanencia en el tiempo. Hay que significar en este aspecto que la importancia de estas mercedes regias no sólo se refiere al plano legal sino también al prestigio que confieren a una iniciativa que al fin y al cabo estaba promovida por particulares. Es natural que desde un primer momento el apoyo de la corona fuese absolutamente fundamental para ellos, lo mismo que la antigüedad de la asociación que habian formado. Todo esto contribuia a enaltecer el significado de la Hermandad para la población y servia para ennoblecerla.

Ahora bien, como ya hemos señalado, en estos primeros tiempos era ante todo una fuerza armada, cuyo impulso partía de los hombres de la ciudad que tenían intereses en los montes y estaban consolidando la propiedad de explotaciones fijas. Pero, sin embargo, los más de sus efectivos necesariamente tenían que ser los que trabajaban en esos mismos montes, generalmente al servicio de las explotaciones mencionadas. En estas condiciones, no es de extrañar que tanto los primeros ordenamientos como muchos de los acuerdos tomados después busquen, antes que nada, asegurar la participación de todos en el esfuerzo hermandino, imponiendo para ello multas a los que no lo hiciesen, y además dedican gran atención a la disciplina de dicha hueste ${ }^{22}$. Claro que si los hermanados eran propietarios de colmenas, como ya hemos dicho, esa calidad otorgaba a su asociación unas características muy claras que casi podríamos llamar gremiales.

\section{LA ORGANIZACIÓN DE LA HERMANDAD DE TOLEDO}

En los dos últimos decenios del siglo xIV, cuando los alcaldes salientes entregaban a los entrantes el archivo de la institución, identificaban siete privilegios en los cuales se condensaba todo lo que los soberanos habían otorgado desde los tiempos de Fernando IV. Estos siete privilegios eran los siguientes: dos confirmaciones de Alfonso XI dadas en 1315, durante su menor edad, y en 1327, una vez llegado al gobierno efectivo del reino, incluyendo los cuatro diplomas concedidos por Fernando IV en los que se ponian las bases que habrían de sustentar jurídicamente la Hermandad en el porvenir; también el de 13 de marzo de 1348, que recogía uno de 1338 y daba seguro a los hermanados; dos cartas de Pedro I, ambas procedentes de las Cortes de 1351, dando valor al anterior y renunciando a pedir a los toledanos "ballesteros apartados", pues ya servían al rey como vecinos de la ciudad; finalmente, las confirmaciones generales de Enrique II y Juan I fechadas respectivamente en 1371 y 1379.

${ }^{21}$ B.N. mss. 13030, fols. 2r-6r, inserto en la confirmación de Alfonso XI de 10 de octubre de 1315, publicado por A. Benavides, Memorias de Fernando IV de Castilla, Madrid, 1860, II, n. 243 y por L. SuÁREZ FERNANDEZ, op. cit., pp. 62-63.

${ }^{22}$ Todavía en 1361 se insistía para que los colmeneros que custodiaban las explotaciones tuviesen permanentemente armas, B.N. mss. 13030 , fol. $122 \mathrm{r}-\mathrm{v}$. 
A estos documentos reales hay que sumar la carta de la ciudad de Toledo dada en 1300 y otros dos escritos referentes al cobro de la asadura - es decir, la renta que sustentaba económicamente el esfuerzo hermandino- sobre los ganados que en su discurrir trashumante se encaminaran a cruzar el Tajo por el puente de Montalbán, así como los contratos de adquisición de algunas propiedades. Por entonces el pequeño archivo del instituto toledano no reunia más que estos fondos y dos libros, el que nos sirve como fuente principal para este artículo y otro más antiguo ${ }^{23}$. Así pues, las pertenencias documentales de la hermandad era bien escasas, pero si hemos reparado en ellas es porque nos permiten comprender lo que para los hermanos era fundamental, esto es, el reconocimiento de la institución por parte del municipio de su ciudad, por supuesto los privilegios reales y también la cobranza de la asadura, así como las escrituras que testimoniaban la formación del patrimonio que iban constituyendo y los dos libros que, con todas las limitaciones que se quiera, reflejaban el funcionamiento de la corporación y sus principales ordenanzas.

La parquedad administrativa de aquel tiempo queda aún más clara si se tiene en cuenta que además de este pequeño conjunto documental la organización apícola no contaba más que con una pesas de hierro para la carne, el sello y un arca hecha en pino y pintada con los castillos y leones de las armas reales, mientras que en 1416 se menciona otra arca de nogal más grande.

Pasada la frontera secular e iniciados los años del cuatrocientos hay que añadir otras dos cartas reales reconociendo que los oficiales de la hermandad fuesen ciudadanos de Toledo y protegiendo las heredades que estos mismos tenían en los montes, más la oportuna confirmación dada por Juan II en su minoría y nuevos papeles sobre el cobro de la asadura de los travesíos. De todos modos, lo que destaca a medida que entra el siglo - concretamente en 1416- es que los fondos estaban empezando a crecer, añadiéndose a los anteriores varios escritos que aluden a la configuración interna de la institución, sobre los que tendremos ocasión de hacer alguna referencia más adelante.

Al frente de la hermandad había dos alcaldes con funciones ejecutivas y judiciales. Se elegian en Navas de Estena, una vez concluida la junta general de las tres hermandades, y aunque con varias excepciones, permanecian en el cargo dos años. Llevaban salario - que por disposición de 1395 era de $150 \mathrm{mrs}$.- y actuaban desde la ciudad. Conocemos la identidad de varios de ellos, y al ver sus nombres inmediatamente se observa que muchos repitieron en el cargo. Es el caso de Domingo Roiz, ya desde el primer momento, pues es uno de los tres hombres buenos reconocidos en 1300 por la ciudad de Toledo para dirigir la naciente Hermandad, y a los pocos años, en 1308, vuelve a aparecer al frente de la misma. Después, a partir de los años sesenta de este mismo siglo, tenemos noticia de 24 individuos citados como alcaldes, de los cuales diez lo fueron en más de una

23 En 1360 el Cabildo de la Hermandad toledana había acordado el traslado de los ordenamientos que entonces existian a un cuaderno de pergamino, B.N. mss. 13030 , fol. 122r. 
ocasión, la mitad de ellos hasta tres veces, y por supuesto, es muy fácil encontrar a estos mismos personajes ocupando otros cargos de la institución. Así que ésta funciona en manos de un grupo de ciudadanos toledanos no muy grande, que encuentran en ella un instrumento que si, por una parte, era eficaz para el ejercicio de la propiedad en la tierra, por otra, podia dar también prestigio y presencia en la vida pública de la urbe.

Por debajo de los dos alcaldes, el organigrama hermandino presenta a los llamados nueves, más tarde denominados regidores. Su actividad consistía en apoyar a los alcaldes viendo con ellos los diferentes asuntos, aunque nunca aparecen en actos judiciales. En el juramento que hicieron en 1382 los dos hombres llamados al ejercicio de la máxima responsabilidad hermandina, se señala que habrian de tener consejo con hombres sabedores, sin duda de cara a los procedimientos judiciales, y en general con los nueves. Para la gestión de los dineros había mayordomo desde fines del siglo xIV, por supuesto se contaba con escribano y también había portero para hacer llamamientos, convocatorias, llevar mensajes, etc.

En el campo actuaban cinco cuadrilleros que son los que verdaderamente articulan la presencia de la Hermandad en el territorio. Su ejercicio duraba dos años aunque muchos de ellos permanecian mucho más y al principio no habia plazo fijo. Cuando apresaban a algún malhechor tenian que trasladarlo a San Pablo de los Montes o a Villarta, si el delito se había producido al sur del río Guadiana, y estaban obligados a avisar inmediatamente a los alcaldes en Toledo para que con prontitud acudiesen a juzgarlo. Se insiste mucho en la rapidez, tanto al mandar el aviso a los alcaldes como al ir éstos a cumplir con sus obligaciones judiciales, porque para la Hermandad la imagen de ejecución rápida y dura de la justicia tiene una importancia capital y se reiterará no sólo ahora sino una y otra vez a lo largo de siglos. Cierto es que esta forma de actuar presenta muy poca flexibilidad y sólo permite llevar a término un número limitado de casos, de suerte que con el tiempo se cambiará por completo, centralizando la actividad judicial en la ciudad.

El verdadero centro de la Hermandad toledana era el cabildo que por entonces se reunía a llamamiento del portero en la iglesia de San Bartolomé de fuera de los muros, con más frecuencia, o bien en muy diversos lugares que van desde diferentes iglesias - Santa Justa, San Isidro del Arrabal, Santiago, etc.- - a la puerta de la catedral o alguna capilla de su interior, pasando por casas de hermanos, etc. ${ }^{24}$. Se hacía una reunión de carácter general el miércoles de cuaresma, luego había comida para los hermanos al día siguiente, y otra también general en agosto para preparar la asistencia a la junta. Claro que además había muchas otras convocatorias cuando había temas que discutir. Lógicamente, debían asistir los alcaides y los nueves, junto con todos los demás hermanos, pero una ordenanza de 1413 poniendo pena a los que no fuesen indica que como en todas las instituciones de la época las ausencias no eran raras. Decimos que el cabildo era el ver-

${ }^{24}$ Es sabido que en tiempos de los Reyes Católicos la Hermandad construyó un edificio específicamente dedicado a sede de la institución y cárcel. Todavía hoy se conserva a espaldas de la catedral. 
dadero núcleo de la asociación apícola toledana porque en el mismo se trataba todo lo importante y se aprobaban las ordenanzas internas destinadas a regir la vida de la entidad y que tanto influían sobre los territorios en los que ésta ejercía su jurisdicción.

Ya sabemos que al unirse los hermanos de Toledo y de Talavera en el otoño de 1300 acordaron reunirse cada año en los montes y lo mismo volvió a repetirse cuando a los dos años se adhirieron los apicultores de Villa Real. El lugar elegido era Aliseda de Estena y la fecha si en principio fue el comienzo de agosto luego se fijó en el primer domingo de septiembre. La junta tenía una importancia muy grande porque era el único elemento de coordinación entre las tres ramas de la institución, aunque en los primeros tiempos era por encima de todo alarde de hombres armados, es decir, exhibición de los efectivos que los hermanados podian movilizar para enfrentarse a las bandas de golfines. Al mismo tiempo, la junta incluía también ritos religiosos y tenía algo de fiesta, de modo que los toledanos pagaron en 1376 por su presencia a un clérigo y un santero ${ }^{25}$, un juglar y también al escribano y el portero del cabildo.

La asistencia tenía que ser por tanto muy numerosa y era una de las principales obligaciones de los asociados, que si no iban tenían que pagar una sanción cuya cobranza se hacía mediante arrendamiento. La dificultad de reunirlos a todos y la abundancia de personas que eludían la carga que representaba acudir durante varios días a la junta, llevó a limitar el número de personas, pagando a todos ellos con cargo a los fondos de la Hermandad ${ }^{26}$. En el caso de Toledo, que al fin y al cabo es el que aquí nos interesa, se hizo así en $1389^{27}$ y después en los años subsiguientes, aunque a finales del siglo el número de asistentes fue variando - doce de a caballo, cada uno con un lancero, y cuarenta ballesteros de los montes en 1394 y al año siguiente veintiocho de a caballo, igualmente con un lancero cada uno, cincuenta ballesteros del monte y seis peones de la ciudad-. Los toledanos convocaban a los que habían de ir a la junta en una reunión que se celebraba en la ciudad en agosto, y como todo eran problemas, en 1405 se decidió comprobar si los de a caballo llevaban las armas y hombres que debian, al tiempo que se hacia jurar a los cuadrilleros para que efectivamente encabezasen a los ballesteros de su cuadrilla. Sin embargo, en lo sucesivo siguieron las dificultades para asegurar la presencia armada hermandina en las juntas, y en 1413 había que volver a ordenar que nadie pusiese excusa y todos los elegidos para ello se pusiesen en marcha para ir a la llega.

Ante este cúmulo de dificultades, en el transcurso de la junta de 1409 se aprobó que se pudieran enviar procuradores en representación de cada una de las tres organizaciones, notificándolo previamente a las otras dos ramas. Lo cierto es

${ }^{25}$ En la junta de 1394 dieron al santero cincuenta mrs. para una bestia.

${ }^{26}$ Lo cierto es que las sanciones no siempre se podían cobrar, a veces se perdonaban y hasta en 1388 se llegó a eximir de la obligación de acudir a las llegas al Monasterio de Sisla, importante poseedor de colmenas.

${ }^{27}$ B.N. mss. 13030 , fols. 123r-24v. 
que este procedimiento, mucho más fácil y barato al no exigir la movilización de grupos de gente armada, se había empezado a emplear tiempo antes - los de Toledo lo hicieron en 1392, por ejemplo- y en aquella misma asamblea de 1409 los de Villa Real estaban representados únicamente por dos procuradores. Pero aunque el nuevo método aparecía como excepcional ${ }^{28}$, no dejaba de ser el primer paso de un proceso que se desarrollará a lo largo del siglo xv y en el cual la asistencia mediante procuradores será cada vez más frecuente. Luego la junta se fue dejando de celebrar algunos años ${ }^{29} \mathrm{y}$, al mismo tiempo, se dejó de utilizar el lugar tradicional -Navas de Estena- haciéndose en diferentes puntos sin que ninguno volviera a tener carácter fijo. En definitiva, la llega perdió su carácter armado y de manera paulatina la irregularidad se fue imponiendo en una evolución que a la larga llevaba a la completa separación de las tres entidades unidas por los acuerdos de principios del siglo XIV ${ }^{30}$. Por otra parte, esta transformación supuso además que actos tan importantes para la Hermandad toledana como la elección de los alcaldes empezaran a hacerse en la ciudad y no en los montes, aprovechando la reunión de los asociados, como se venía haciendo tradicionalmente.

No obstante, en los años del trescientos y todavía durante cierto tiempo en la centuria siguiente, eran muchos los que iban a las juntas y se planteaban problemas de abastecimiento. Para resolverlos se recurrió muy pronto a un procedimiento muy del gusto de la época, contratando el aprovisionamiento de algunos productos más necesarios a ciertas personas en régimen de monopolio, es decir, sin que ningún otro pudiera llevarlos. Se quería así garantizar la oferta de esos alimentos a precios fijados en el acuerdo, animando la contratación mediante algún dinero que se ofrecía a los abastecedores por asumir el compromiso. Este es un procedimiento muy habitual en la Baja Edad Media, utilizado con particular frecuencia por los concejos para dar respuesta a la demanda urbana. Por lo tanto no es sorprendente que las hermandades lo utilizaran tempranamente. Así pues, tenemos noticia de la contratación de la carne desde 1377, entre 25 y 30 carneros cada junta a los precios de la ciudad de Toledo, añadiéndose una ternera desde el final del siglo. A partir de 1398 de aseguró de esta manera la cebada y la paja, y más adelante, a mediados del siglo $\mathrm{x}$, sabemos que también el pan era objeto de contratos de esta clase ${ }^{31}$. Pero volviendo a los datos del siglo XIV interesa hacer notar que la cifra de carneros consumidos en cada ocasión da idea de la convocatoria de

28 En 1423, por ejemplo, las hermandades de Toledo y Talavera acudieron por cabildos y caballerías, como se decia entonces, es decir, según lo tradicional, mientras que la rama manchega lo hacía por procuradores, A. M. Talavera Sta. Hermandad, leg. 1, n. 2.

${ }^{29}$ En 1439 no se celebró la llega y las tres hermandades intercambiaron cartas en el mes de agosto confirmando la continuidad de su asociación, A. M. Talavera, Sta. Hermandad, leg. 1, n. 7. El año anterior se debatió mucho entre ellas sobre el modo de hacer la junta, por razón de la pestilencia que repercutia al menos sobre Toledo y Ciudad Real, de modo que muchos hermanos habian abandonado sus residencias, otros estaban enfermos y muchos pasaban arduos trabajos en sus casas, A. M. Talavera, Sta. Hermandad, leg. 24, n. 8 , leg. 23, n. 1 y leg. 23, n. 8.

30 He resumido este proceso de decadencia de la junta anual a partir de los años veinte del siglo xV en mi Santa Hermandad Vieja..., cit., pp. 66 y ss.

${ }^{31}$ Santa Hermandad Vieja..., cit., p 82. 
una tropa numerosa cuya presencia a día fijo en los montes adquiría una dimensión claramente disuasoria.

Las juntas anuales no sólo se hacian con los hombres de las tres hermandades, como hasta ahora hemos ido viendo, sino que además en todas las que tenemos registradas a lo largo del siglo xIV y principios del xv también aparecen procuradores de varias localidades del entorno toledano: Puebla de Alcocer, Herrera, Fuenlabrada, Pulgar, Yébenes, Marjaliza, Puebla de Montalbán y Cuerva, además de Chillón, situada al oeste de la demarcación de la Hermandad de Villa Real. Quiere esto decir que los propietarios de colmenas de estas localidades estaban adheridos a la iniciativa hermandina y por eso, dependiendo de la organización toledana, acudían con sus representantes a las juntas. En el caso de Chillón la dependencia tenía lugar con respecto a la Hermandad manchega, siendo una suerte de apéndice de la misma, del mismo modo que las anteriores lo eran de la toledana.

En 1404 representantes de Puebla de Alcocer se presentaron en Toledo para solicitar el retorno a la Hermandad de los hombres buenos de esta localidad que tenian intereses en los montes. No sabemos el motivo que había propiciado su separación, pero estos años conforman un periodo de ajuste en el discurrir de la institución, durante el cual las diferencias internas salen a la luz imparablemente. Lo cierto es que los vecinos de este núcleo invocaban su pertenencia anterior y fueron acogidos de nuevo sin mayor dificultad, sólo que al hacerlo se anotó en el libro que nos sirve de fuente principal la formula mediante la cual la institución hermandina funcionaba en este lugar $y$-hay que suponer- en las otras poblaciones vinculadas a aquella. El texto utiliza la expresión cuadrilla porque eso es lo que en realidad iba a ser el ámbito de Puebla de Alcocer dentro del sistema orgánico de los colmeneros. Por consiguiente, el cuadrillero era el elemento esencial, con una doble finalidad que era la persecución de malhechores, por una parte, y la convocatoria de los hombres de la cuadrilla, por otra. Naturalmente, de las sentencias se ocupaban los alcaldes y, una vez pronunciadas, el cuadrillero era el encargado de proceder a la ejecución de los castigos, en algún camino frecuentado para asegurar la ejemplaridad. Por lo que se refiere a la junta general era obligatoria la presencia de este personaje, acompañado por cuatro hombres de la zona, para dar cuenta de todo lo acontecido a lo largo del año en su demarcación.

De este modo, la Hermandad de Toledo, impulsada desde la ciudad como hemos visto, actuaba en los Montes de la misma - comprados por la urbe en 1246organizando el territorio en cinco cuadrillas durante el siglo XIV. Dichas cuadrillas eran las de Estena, Herrera, Bullaque, Guadiana, y Arroba y Alcoba ${ }^{32}$, más el ám-

32 Aunque con el paso del tiempo este número varió y en un documento de 1458 (A. M. Talavera, Sta. Hermandad, leg. 1, n. 3) se habla de nueve: Milagro, Navas de Estena, Arroba, Puerto Marchés con San Pablo, Santa María de Herrera con Cedenilla, Santa María del Águila con Peñaguilera, Pulgar, Santa Maria de la Nava con Marjaliza y San Andrés con Yébenes, pero es fácil observar que varias de ellas se refieren a algunos de los pueblos referidos anteriormente. Sobre el ámbito de los Montes de Toledo en general es interesante consultar la obra de J.-P. MoLÉnAT, Campagnes et monts de Tolède du XIle au 
bito montaraz de cada uno de los pueblos que hemos indicado antes, cuya articulación devendrá semejante a una cuadrilla por cada uno de ellos. De esta forma, limitaba por el oeste con La Jara talaverana, jurisdicción de la Hermandad de esta villa, y por el sur con los hermanos de Villa Real, cuya acción se extendía por las tierras pertenecientes a la Orden de Calatrava.

Estructurada de la manera que hemos estudiado, y teniendo siempre como centro la ciudad de Toledo, había en la Hermandad una contradicción ineludible entre los ciudadanos y los apicultores que vivian en el ámbito rural, reducidos a un segundo plano casi carente de significación en el organigrama hermandino. Bien es verdad que sobre esta contradicción se había edificado la entidad toledana, pero eso no impidió la protesta de los que estaban en una posición más desfavorecida, y lo hicieron con tanto impetu que su voz llegó hasta el trono. No obstante, la decisión que al respecto se tomó en la corte durante la minoría de Juan II - teniendo como fundamento el que la Hermandad se habia constituido a partir de los ciudadanos-presenta un carácter profundamente conservador ${ }^{33}$. La dirección suprema seguía en manos de dos hombres buenos de la urbe, en ella deberian conservarse los privilegios que sustentaban la institución y también el mayordomo habría de ser toledano. Es decir que la ciudad seguía siendo el eje y los hermanos que en ella vivían los únicos con posibilidad de acceder a los puestos principales. Por lo tanto, se venía a confirmar en su totalidad el esquema de funcionamiento utilizado hasta entonces y tan sólo se observa una pequeña concesión al admitir que tres hombres de procedencia rural estuviesen presentes a la hora de tomar la cuenta del mayordomo.

Los propietarios de la ciudad habían salido ganando indudablemente, pero debe tenerse en cuenta que estas disputas no se refieren sólo al protagonismo de unos y otros dentro de la jerarquía hermandina. En realidad, el enfrentamiento muestra una amplitud mucho mayor y afecta en general a las posesiones que los ciudadanos tenían en el territorio dependiente de Toledo. Sin duda hay que entender que lo que se dilucidaba era la proyección sobre la tierra de los propietarios rurales frente a los urbanos. Por eso éstos últimos se preocuparon también de conseguir cartas reales -fechadas en 1405 y 1408-que aseguraban no sólo las posadas de colmenas sino también sus heredades, viñas, molinos, etc. ${ }^{34}$. Sólo con

\footnotetext{
XVe siècle, Madrid, 1997, particularmente parte II, capitulo 2, pp. 215 y ss. Conviene tener en cuenta también la Hermandad formada por Mora, Orgaz, Ajofrín, Almonacid, Mascaraque, Villaminaya, Manzaneque, Arisgotas, Casalgordo, Mazarambroz, Sonseca, Villaseca de la Sagra, Pulgar y Layos, alrededor de una dehesa común a todos ellos. Aunque después su nombre será otro, en tiempos de los Reyes Católicos se llamaba Hermandad Vieja de las dos Sislas Mayor y Menor de Tajo aquende y tenia funciones de justicia en despoblado. Ver al respecto J. M. SÁNCHEZ BENITO, "Consideraciones sobre la Hermandad de San Martín de la Montiña (Siglos XIV-XV)", Anales Toledanos, XXV, 1988, pp. 47-56.

${ }^{33}$ La fecha del documento es 16 de mayo de 1407, B. N. mss. 13030, fol. 134r, publicado por L. SuÁREz Fernández, op. cit, pp. 69-70.

34 Los documentos de 1405 y 1408 no se conservan y tan sólo se conocen por las referencias contenidas en el libro de la Hermandad que estamos siguiendo como fuente principal al relacionar las escrituras que la institución conservaba a principios del siglo xv y pasaban de un mayordomo a otro cuando se renovaba el cargo.
} 
las garantías que los hombres de la ciudad consiguieron mediante todos los mandatos regios que hemos citado, se procedió a renovar la estructura interna de la Hermandad, haciendo expresamente acto de recepción en ella de los hombres buenos de la tierra de Toledo y, más en concreto, señalando que los de determinados lugares - Cuerva, Gálvez, Jumela, Menasalbas y Pulgar- acudiesen a la junta anual de las Navas de Estena. Lógicamente, hemos de que entender que la reintegración en la organización de los de Puebla de Alcocer, aunque algo más temprana, se produce en el marco que estamos mostrando.

En consecuencia, hay que aceptar que la asociación hermandina no es sólo el resultado de la iniciativa de los vecinos de Toledo que tenían intereses en los montes, encabezando a aquellos que trabajaban en sus explotaciones, sino que tiene mayor complejidad. Incluye también a hombres buenos de los pueblos de la tierra, igualmente implicados en la explotación apícola de los baldíos que hubiera en el ámbito de cada una de esas poblaciones, y tiene por lo tanto una estructura triple, en la que forman ciudadanos, dependientes de éstos y vecinos de los pueblos, ocupando cada grupo un lugar determinado ${ }^{35}$.

Sin embargo, no terminaron aquí los conflictos. Poco tiempo después, había fuertes divisiones en el cabildo toledano y se hablaba de ligas de gentes en su interior. Por lo que sabemos, el alcalde Pedro Fernández de las Cuevas, que encabezaba uno de los bandos, tenía tal oposición que muchos se querellaron contra él y la cuestión llegó a la junta anual, implicando a las otras hermandades. Como consecuencia de las querellas el alcalde toledano fue prendido por los hermanos de Talavera, pero sus partidarios lograron mandamientos en su favor de las autoridades urbanas de Toledo y no contentos con eso le sacaron por fuerza de la prisión en que estaba, al mismo tiempo que por su parte presentaban denuncias e intentaban apresar a sus contrarios en el cabildo. Queda claro, en conclusión, que la Hermandad durante los primeros años del siglo $x \vee$ pasaba por dificultades que afectaban seriamente a su cohesión, primeramente por la relación desequilibrada entre los hermanos de la ciudad y los del medio rural, y más tarde por razón de disputas por el poder, o lo que es lo mismo, por los cargos directivos, que es lo que se ventilaba en estos enfrentamientos cuyos protagonistas son personajes de indudable relieve. Todos ellos habían ocupado los diferentes puestos de dirección de la Hermandad y, sin duda, contaban con no poca proyección en la ciudad.

También en la Hermandad manchega hubo toda suerte de choques, tanto en la primera mitad del siglo xv como en sus años finales, y habrá que entender que al igual que ocurría en ella la ínstitución toledana no sólo sufría las diferencias que se deducian de su propia estructura sino también las derivadas del ambiente sociopolítico. Finalmente, los problemas que se suscitaron alrededor de Pedro Fer-

${ }_{35}$ Para entender bien esto hay que tener en cuenta además que una cosa eran los Montes de Toledo, comprados por la ciudad en el siglo XIII, y otra la tierra dependiente de la jurisdicción urbana. 
nández de las Cuevas se zanjaron en $1417^{36}$ de manera favorable a sus oponentes, que consiguieron el apoyo de las otras dos hermandades, pero al cabo de un cierto tiempo los mismos temas volvieron a resurgir, conjugándose ahora el enfrentamiento por los puestos dirigentes y las diferencias entre ciudadanos y rurales. Al parecer algunos hermanos para apoyar sus ambiciones buscaron el apoyo de los que vivían fuera de la ciudad, recurriendo a los primeros privilegios y ordenamientos de la institución y negando validez a los hechos con posterioridad. Una vez más, el choque -que conocemos por dos cartas dirigidas a los talaveranos ${ }^{37}-$ dividió profundamente a los hermanos y llegó a presentarse en la junta general del año 1433.

\section{RECURSOS ECONÓMICOS Y FUNCIONALIDAD DE LA HERMANDAD}

Se sabe que la financiación de toda la organización hermandina descansaba sobre una renta específicamente destinada a este fin que era la asadura. Consistía en un gravamen sobre el paso del ganado, en la relación porcentual de una res por cierto número de cabezas ${ }^{38}$, que ya aparece en el segundo de los privilegios reales obtenidos por los hermanos, fechado en 1303. Lo que ocurre es que en el tenor de este documento regio no se procede a la creación de esta renta sino que se da por entendida su existencia, de forma que entre las peticiones dirigidas al soberano se decía que al pedir las asaduras a pastores y vaquerizos no se las querían pagar y la corona ordenó que efectivamente lo hicieran. El origen de esta renta se confunde entonces con el de la misma Hermandad, aunque lo más probable es que si en las primeras correrías contra los golfines hubo alguna forma de colaboración entre ganaderos y colmeneros, y de ahí se iniciaron los primeros pagos, en la medida en que las huestes hermandinas defendian a cuantos utilizaban el monte, muy pronto hubo disconformidades entre ambos. Lo demuestra la carta de la ciudad de Toledo de octubre de 1300 - ya cintada anteriormente-, donde ya se denunciaba que los pastores negaban su ayuda contra los golfines, y lo mismo el acuerdo hecho un mes más tarde entre los hermanados de Toledo y de Talavera en el que se vuelve a insistir para que también los ganaderos fueran contra los golfines.

Los dos privilegios siguientes, otorgados por Fernando IV, vuelven a insistir en la obligación de pagar la renta que sustentaba a la Hermandad y lo mismo los de Al-

36 Los documentos que nos permiten aproximarnos a este asunto son el acta de la junta extraordinaria que para tratar del mismo reunió a representantes de las tres hermandades en octubre de 1416, A. M. Talavera, Sta. Hermandad, leg. 1, n. 3, y dos cartas cruzadas entre toledanos y talaveranos en mayo de 1417, A. D. Toledo, leg. 34, n. 2.

37 Ambas cartas están fechadas el 28 de agosto y el 25 de septiembre de 1433 y se conservan en el A. M. Talavera, Sta. Hermandad, leg. 24, n. 8.

38 He tratado con anterioridad sobre esta renta al estudiar las tres hermandades en mi libro Santa Hermandad Vieja..., cit., pp. 197 y ss., y en comparación con otros gravámenes locales sobre la trashumancia ganadera en P. GaRcia MARTíN Y J. M. SÁNCHEZ BENITO, "Arbitrios locales sobre la propiedad semoviente en Castilla durante los siglos XIV y Xv", En la España Medieval. Estudios en memoria del profesor D. Claudio Sánchez Albornoz, I, 1986, pp. 399-411. 
fonso XI, tanto de la etapa de su minoría como al llegar a la mayoría de edad. El siguiente documento real, fechado el treinta de marzo de 1327, se dedicó exclusivamente a esta temática, extendiendo la obligación tanto a los porquerizos como a los merchanes ${ }^{39}$, y finalmente, en 1338 , tras crearse el servicio y montazgo, los hermanos consiguieron que su principal fuente de financiación quedase a salvo sin ser absorbida por la nueva renta real ${ }^{40}$. Quedaba así configurada una imposición que habría de perdurar largo tiempo, como verdadero cimiento de la triple institución, cuya extensión alcanzaba a los ganados, tanto vacunos como ovejunos, bien sean cabañiles, travesíos o merchaniegos y también, como hemos visto, a los puercos.

En cuanto a la praxis de su cobranza, aunque había sido otorgada mancomunadamente a las tres corporaciones apícolas, desde un primer momento se acordó un reparto territorial, correspondiendo los ganados que vinieran por la Cañada Segoviana a los talaveranos, la Cañada de Cuenca a los hermanos de Villa Real y, por último, la Cañada Toledana a los de esta ciudad ${ }^{41}$; es decir, cada hermandad cobraba sobre el tráfico pecuario que discurriera por su demarcación. En este marco, los toledanos tenían su principal punto de recaudación en el puente de Montalbán, aunque por documentación más tardía sabemos que también actuaban en El Molinillo y San Andrés de Yébenes ${ }^{42}$. Por lo general, el cobro se hacía mediante arrendamiento, según era normal en las haciendas de la época ${ }^{43}$, y en cuanto a los que por este medio obtuvieron la cobranza de la renta durante el siglo XIV solamente decir que con frecuencia eran hermanos y solían actuar formando pequeñas sociedades.

Durante la mayor parte del siglo XIV eran los alcaldes los que se ocupaban de los dineros de la hermandad toledana y, por consiguiente, ellos eran los que tenían que presentar las cuentas. Con frecuencia lo hacía cada alcalde por su lado y en una fecha de fines de septiembre o principios de octubre. Sin duda, la irregularidad era notoria, al punto que no es raro el retraso durante mucho tiempo del cierre de las cuentas, a veces se presentaban a la vez las de varios años y en otras ocasiones eran ingresos concretos los que se declaraban y no la totalidad. En fin, este panorama de desorden administrativo no puede sorprendernos demasiado porque se da a menudo en distintas instituciones de esta misma época y además ya hemos visto lo escaso del aparato administrativo a disposición de los hermanos.

En 1396, al tomar las cuentas anteriores, se decidió que el escribano tuviera cargo de ellas a cambio de soldada de doscientos mrs., y al año siguiente se nom-

${ }^{39}$ A.H.N. Códice 818B, fols. 24v-26v, publicado por J. M. SÁnchez Benito, Santa Hermandad Vieja..., cit., n. 2 .

${ }^{40}$ A.H.N. Diversos, leg. 1, n. 2 y A. M. Talavera, Sta. Hermandad, leg. 25, n. 57.

${ }^{41}$ Conocemos este acuerdo por un documento de 1334, a su vez inserto en otro del año siguiente, por el cual las tres hermandades se pusieron de acuerdo para reclamar el derecho de los hermanos de Villa Real, a los cuales la Orden de Calatrava impedía cobrar la asadura, A.H.N. Códice 818B, fols. 32v-41r.

42 J. M. SÁnCHez Benito, Santa Hermandad Vieja.., cit., p. 204.

43 Hay un acuerdo considerablemente antiguo, de 1306, para que no se perdonase ninguna cantidad a los arrendadores y para que ninguno de la Hermandad rogase en su favor, B.N. mss. 13030 , fol. $118 \mathrm{v}$. Se renovó en 1367, lbídem., fol. $122 \mathrm{v}$. 
bró un mayordomo, Pedro Fernández de las Cuevas, que actuó en el ejercicio 1397-98, responsabilizándose inmediatamente de su gestión. Poco a poco se empieza a regularizar la rendición de cuentas año por año y ante unas personas determinadas que son los alcaldes y nueves, más otros hermanos que también pudieran acudir. Se observa por tanto una tendencia encaminada a un mayor orden que es muestra de creciente maduración pero que probablemente se vio facilitada por circunstancias momentáneas. Efectivamente, el excedente que presentaba el balance de 1395, en manos de los alcaldes salientes, no se entregó puntualmente a los nuevos responsables de la institución y además el procedimiento se complicó al morir uno de ellos dejando un menor que lógicamente tuvo que asumir la deuda. Probablemente las dificultades consecuentes a este hecho convencieron a todos de la necesidad de contar con una persona específicamente dedicada a los dineros.

El mismo desorden que hemos venido señalando, así como el que no haya más que una contabilidad mínimamente detallada en el libro que estamos utilizando como fuente principal, todo ello, dificulta mucho el análisis evolutivo de la capacidad económica que presenta la agrupación toledana. Nuestros datos muestran cifras de ingreso y gasto, así como el saldo de la mayoría de los años comprendidos entre 1377 y 1418, pero como ha quedado indicado anteriormente estos datos se refieren a veces a varios años y no siempre se puede saber si el dinero excedentario del año anterior -el alcance - se incluye como ingreso al siguiente. Así las cosas, lo que se puede afirmar es lo siguiente: En primer lugar, todos los balances son positivos sin excepción alguna, de modo que la Hermandad tiene una hacienda sana que le permite funcionar con holgura. En segundo término, y por lo que se refiere a los ingresos, se nota que desde el comienzo del siglo xv hay un incremento que se acentúa claramente a partir del ejercicio 1406-07; si bien, hay que tener en cuenta que en estos años del cuatrocientos parece que casi siempre se incluye el alcance anterior como ingreso. Por último, el gasto sigue los mismos derroteros aunque creciendo de forma algo más moderada.

Ya hemos dicho que los dineros procedian principalmente de la asadura, particularmente de los ganados trashumantes que pasaban por el puente de Montalbán. Por lo tanto, se entiende a la perfección el interés de los hermanos por todo lo que se refiriera a la confirmación de los privilegios y, en general, a la defensa de su renta. En 1398 el arrendador declaraba haber perdido $1900 \mathrm{mrs}$. al venir menos ganado por la fuerza que Gómez Fernández Palomeque hacía a los pastores, y consecuentemente en el ejercicio 1398-99 se observa un cierto descenso en las entradas que registran las cuentas.

Hubo, por otra parte, problemas entre las hermandades, aunque se solventaban internamente recurriendo al arbitraje de aquella de las tres que no tuviese relación con el asunto en litigio ${ }^{44}$. De esta manera quedó resuelto el pleito que hacia

${ }_{44}$ En la junta general de 1412 se revalidó el acuerdo ya existente para que en el caso de pleitos entre las tres hermandades se solventasen del modo que hemos indicado, sin mayores complicaciones pro- 
1411 se suscitó entre los de Toledo y Villareal por razón de los ganados travesíos, dando sentencia los talaveranos con la aprobación de todas las partes, y lo mismo volvió a repetirse en 1416 cuando al caer el puente de Montalbán los rebaños se desviaron hacia Alcolea, penetrando entonces en la zona de influencia de Talavera. Como éstos no lo dudaron y procedieron a la recaudación, en Toledo se sintieron perjudicados y fueron los alcaldes manchegos los que tuvieron que zanjar el caso, de forma, por cierto, bastante favorable a los últimos ${ }^{45}$.

Otro ingreso regular, mucho menos importante, es el pago que los propietarios de colmenas tenían que hacer, en función del número que tuviesen, por su pertenencia al instituto. Este ingreso consta sin la menor duda e incluso se observa que en el siglo XIV se arrendaba, pero por lo que sabemos no aparece en las otras dos ramas de Talavera y Villa Real, y debió acabar desapareciendo. De todos modos se disponía de otras fuentes de renta cuya constitución habia hecho posible el superávit que siempre se repetía al final de cada uno de los ejercicios. Efectivamente, en 1373 sabemos que se sacaba beneficio del arrendamiento de la posada de colmenas llamada de Nuño Román, que era propiedad de la Hermandad, y al año siguiente se compraba la de la Canaleja por $3200 \mathrm{mrs}$. Después, en el trienio 138890 hubo alguna otra compra de colmenares y en 1398 los responsables de la entidad declaran tener además las de Navacerradilla, El Hoyo y El Castaño ${ }^{46}$. De forma que la Hermandad, que al fin y al cabo era una organización de colmeneros, invierte buena parte de sus excedentes económicos precisamente en el sector de la apicultura, formando poco a poco un patrimonio en los montes que con el tiempo se irá complementando con la adquisición de inmuebles en la ciudad, y ya en 1413 se habla de lo que habian rendido las casas que por entonces tenían ${ }^{47}$.

Por lo demás, los ingresos de la institución se completaban en el siglo xiv con las caloñas que principalmente procedian de los miembros de la institución que no iban a la junta anual. Dichas multas muestran el orden que los hermanados

cesales. Esta renovación no podía ser más oportuna porque en aquel momento los institutos de Toledo y Villa Real disputaban por el cobro de la asadura a los ganados travesios. En 1438, cuando en la entidad manchega se disputaba abiertamente por los cargos directivos se recurrió a árbitros comisionados por las otras dos organizaciones hermanas, aunque la crisis era profunda y no se solucionó de manera inmediata, A.M. Talavera, Sta. Hermandad, leg. 23, n. 1, leg. 24, n. 8 y leg. 1 , n. 7.

${ }_{45}$ A. M. Talavera, Sta. Hermandad, leg. 1, n. 3. Sobre este caso ver mi Santa Hermandad Vieja..., cit., p. 205. En cuanto a las reses pertenecientes a individuos pertenecientes o vinculados a alguna de las hermandades había igualmente acuerdo para no cobrarles, al menos parcialmente, si pasaban por los territorios de las otras dos entidades. Parece que en 1423 volvió a haber algún problema por la asadura entre las hermandades de Toledo y Talavera. Sabemos que en septiembre de este año se trató en el cabildo de la segunda sobre una carta de los toledanos, llegada a la villa poco antes, que parece sugerirlo asi. A. M. Talavera, Sta. Hermandad, leg. 1, n. 2.

${ }^{46}$ Sin embargo, no se conserva ninguno de los contratos de compra hasta el que se hizo en 1435 al adquirir la cuarta parte de la posada de Puerto Rubio con 27 colmenas, A.H.N. Diversos leg. $78, n .1$.

${ }^{47}$ De los años posteriores, desde mediados del siglo $\mathrm{xv}$, tenemos la documentación correspondiente a la compra de nueve inmuebles en Toledo y otras nueve posadas de colmenas, J. M. SÁNCHEZ BENITO, Santa Hermandad Vieja..., cit., p. 227. El contrato más antiguo, referente a bienes urbanos, data de 1437 y se refiere a unas casas con bodega en la collación de San Miguel, A.H.N. Diversos, leg. 77, n. 1. Naturalmente, las otras dos hermandades siguieron conductas similares constituyendo igualmente patrimonios inmobiliarios. 
habían forjado para aprestar sus efectivos armados y solían arrendarse. Sin embargo, a veces se perdonaban y en 1375, aun habiendo arrendatario, no se llegaron a cobrar.

En cuanto a los gastos, hay unas partidas fijas que son los salarios - de alcaldes, escribano, portero, clérigo y cuadrilleros-, la remuneración de los que asistían a la junta general y las comidas que tenían lugar el primer jueves de cuaresma y el día que se tomaban las cuentas. Por lo demás, la información suele ser imprecisa y apenas si nos ofrece alusiones a algún pleito en defensa de los derechos de la Hermandad, el coste de la confirmación de privilegios o, en términos muy genéricos, la persecución de malhechores y la pesquisa de fuegos acontecidos en los montes. En cuanto a limosnas, muy pocas y esporádicas ${ }^{48}$.

Para mayor detalle cabe acudir únicamente a las cuentas correspondientes a las anualidades $1374-75$ y 1375-76. Al ser las únicas que reflejan la estructura del gasto, permiten verificar que son las comidas y los costes de la junta general las principales partidas, hasta superar entre ambas la mitad del total. A continuación, los salarios y luego una serie de conceptos de inferior importancia, incluyendo el envío - de acuerdo con la ciudad- de cinco ballesteros para una determinada misión armada, así como algunas deudas, mensajerías, la devolución de una multa y cincuenta mrs. dados para la obra que se estaba haciendo en la iglesia, tan unida a la Hermandad, de San Bartolomé de Fuera en Toledo. Pero lo que más sorprende es que las tareas de persecución del delito, verdadera razón de ser de la institución, y de la asadura que garantizaba su subsistencia, apenas encuentran reflejo contable. Solamente se citan $500 \mathrm{mrs}$. dedicados a la prisión de un cierto colmenero, lo cual supera escasamente el $12 \%$ del total.

Comidas

Gastos de la junta anual

Soldadas de oficiales y cuadrilleros

Prisión de un colmenero

Misión de cinco ballesteros

Deudas de la Hermandad

Devolución de caloñas

Ayuda para la obra de San Bartolomé

Mensajerías
$1134 \mathrm{mrs}$.

1125

620

500

290

253

60

50

33

Pero para terminar lo que se refiere a las cuentas hermandinas digamos que también llama la atención el volumen que muestran las albaquias, es decir, las deudas a favor de la Hermandad derivadas especialmente del impago de los arrendamientos de la asadura y otras rentas de la institución en años anteriores. Es

48 En el capítulo de limosnas, apenas si se registra una referencia en 1393, cuando se dieron treinta mrs. a dos frailes que pedian en la liega, y al año siguiente cincuenta mrs. a cierto santero para adquirir una bestia. 
interesante resaltar que todavía en estos años, 1374-76, se observa el reflejo de las dificultades de la guerra civil, de tal manera que sólo ahora se procedia a la regularización de lo que la Hermandad debía a uno de sus miembros desde el tiempo del cerco de la ciudad y, en sentido contrario, aún quedaban por recibir dineros de la asadura de 1365. Pero, en resumen, la suma de las albaquías no es pequeña, ascendía a $2800 \mathrm{mrs}$., cuando los ingresos se valoraban en 4267 , y por su variedad reflejan una situación de desorganización no pequeña.

Hay muy pocas evidencias en la documentación que estamos manejando sobre la actividad delictiva perseguida por los hermanos, y por lo tanto nada podremos añadir a lo ya dicho en otros trabajos sobre esta cuestión. No obstante, hay un detalle que no debemos olvidar, y es que, entre los poquísimos casos que podemos identificar con las fuentes que estamos manejando, los colmeneros son los protagonistas de más de un crimen. También es verdad que al menos una vez se habla de un grupo de delincuentes, pero parece que la Hermandad a fines del siglo XIV y principios del siglo XV actúa más contra los desmanes que pudieran hacer los propios habitantes de los montes que contra malhechores en grupo, apuntando así en la misma dirección que muestran los estudios que se han hecho con los datos mucho más detallados del cuatrocientos y sobretodo de sus años finales ${ }^{49}$.

Algo más se puede decir acerca de los incendios forestales, que la Hermandad perseguía penalmente con la mayor dureza y trataba de prevenir con todos los medios a su alcance. Se entiende con facilidad que si el fundamento de la institución era la economía apícola, y ésta necesariamente se apoyaba en la disponibilidad de plantas aprovechables por las abejas, por fuerza el deterioro del manto vegetal preocupaba extraordinariamente a los hermanos, cuya reacción no se hizo esperar. Desde muy pronto se procuró limitar la peligrosidad del fuego prohibiendo encenderlo en la época más calurosa del año ${ }^{50}$. A esta medida preventiva se añadió la obligación de acudir a apagarlo todos los que vivieran en los montes, dos leguas alrededor de la quema ${ }^{51}$, y por supuesto, las conductas incendiarias se convirtieron en delito perseguible por los cuadrilleros ${ }^{52}$. No obstante, en 1389 se denunciaba que las llamas quemaban colmenares y hasta personas humanas, y la pena para los responsables se aumentó hasta la muerte, acordando las tres hermandades poner dos hombres cada una para que vigilasen el territorio averiguando quienes eran los culpables ${ }^{53}$. En 1395 se confirmó lo acordado, razonando que el monte era objeto de una verdadera amenaza y con ello quedaba en cuestión la propia

49 Remito a los trabajos de J. M. MEndoza GarRIdo, particularmente, Delincuencia y represión..., cit., y a los míos Santa Hermandad Vieja..., cit., pp. 270-84, “Delincuencia y vida rural...», cit., pp. 67-74 y últimamente la ponencia presentada en el III Coloquio del Centro de Historia del Crimen de Durango y ya citada "Hermandades y delincuencia rural ...", cit.

50 B.N. mss. 13030 , fol. $119 \mathrm{v}$.

51 B.N. mss. 13030 , fols. $121 v-22$ r.

52 En 1385 se revalidó una ordenanza ya existente para que si alguna posada de colmenas se despoblaba por fuego, el causante del mismo pagase en pena seiscientos mrs. B.N. mss. 13030, fol. 123r.

${ }_{53}$ Aunque eran los cuadrilleros quienes realizaban luego las persecuciones y detenciones que fuesen pertinentes. 
existencia de la triple corporación apícola. Al año siguiente se volvía sobre lo mismo ordenando además que los cuadrilleros persiguiesen sin tregua a esta clase de delincuentes con todos los efectivos de su cuadrilla, y otra vez en 1402 se hablaba de muchos y muy grandes fuegos para insistir en la misma normativa.

En conclusión, este fenómeno se manifiesta de una manera estrictamente paralela al crecimiento de la población y, por lo tanto, de la explotación de los zonas montuosas en las que actuaban las hermandades. Lo que muestran los datos que acabamos de exponer es que ya desde fines del siglo XIV había en ellas tendencias de esta índole y que, por consiguiente, estos espacios - y en particular los Montes de Toledo- en gran medida marginales en los procesos repobladores anteriores, estaban cobrando alguna vitalidad. Naturalmente, frente a estas dinámicas poco podía hacer la Hermandad por mucho que utilizara sin la menor vacilación sus recursos humanos y todo su repertorio de medidas preventivas, policiales y judiciales.

Esta temática nos lleva, para terminar, a la vertiente propiamente apicola de la institución toledana. Como asociación de propietarios, la Hermandad fijaba el marco de cada posada de colmenas, es decir, la extensión de monte reservada a cada explotación ${ }^{54}$, y se ocupaba de regular cuestiones que pudiesen suscitar dudas, como los enjambres silvestres o la enajenación de colmenas colocadas en posada de otro, dando en este caso preferencia para su adquisición al dueño de la misma ${ }^{55}$. Por otra parte, se preocupó muy tempranamente por todo lo que se refiere a las relaciones entre los amos, fundamentalmente de la ciudad, y los colmeneros que guardaban sus posesiones. En este aspecto la entidad viene a ser un instrumento para sujetar a estos hombres e imponer las conveniencias de los primeros. Claro que procuraron también aliviar a estas gentes del pago de impuestos, impidiendo a los vinculados a la Hermandad comprometerse en el arrendamiento de monedas, alcabalas y otras rentas que no fuesen los derechos y arbitrios de la ciudad.

En fin, es claro que las tres hermandades, y la toledana en particular, consiguieron adquirir un control muy grande sobre la práctica de la apicultura y sobre la vida montaraz en términos amplios ${ }^{56}$. Además, de esta manera se fue perfilando un ámbito jurisdiccional civil mediante medidas que impedian a las gentes de los montes emplazarse ante las justicias ordinarias, teniendo que llevar sus diferencias ante los cuadrilleros y alcaldes hermandinos ${ }^{57}$.

54 B.N. mss. 13030 , fols. $120 \mathrm{v}-21 \mathrm{r}$.

${ }_{55}$ B.N. mss. 13030 , fol. $120 \mathrm{v}$.

${ }^{56}$ Sobre la apicultura toledana en el ámbito global de Castilla J. M. SANCHEZ BENito, "Datos sobre la producción apícola castellana en la Baja Edad Media», Estudis d'Història Económica, 1989, pp. 11-25 y también Santa Hermandad Vieja..., cit., pp. 289-96.

${ }^{57}$ Ver al respecto mi Santa Hermandad Vieja... cit., pp. 297-301. 